\title{
Regression Methods for Ophthalmic Glucose Sensing Using Metamaterials
}

\author{
Philipp Rapp, ${ }^{1}$ Martin Mesch, ${ }^{2}$ Harald Giessen, ${ }^{2}$ and Cristina Tarín ${ }^{1}$ \\ ${ }^{1}$ Institute for System Dynamics, University of Stuttgart, Pfaffenwaldring 9, 70569 Stuttgart, Germany \\ ${ }^{2}$ 4th Physics Institute, University of Stuttgart, Pfaffenwaldring 57, 70569 Stuttgart, Germany
}

Correspondence should be addressed to Philipp Rapp, rapp@isys.uni-stuttgart.de

Received 31 May 2011; Accepted 5 August 2011

Academic Editor: David Hamilton

Copyright ( 2011 Philipp Rapp et al. This is an open access article distributed under the Creative Commons Attribution License, which permits unrestricted use, distribution, and reproduction in any medium, provided the original work is properly cited.

\begin{abstract}
We present a novel concept for in vivo sensing of glucose using metamaterials in combination with automatic learning systems. In detail, we use the plasmonic analogue of electromagnetically induced transparency (EIT) as sensor and evaluate the acquired data with support vector machines. The metamaterial can be integrated into a contact lens. This sensor changes its optical properties such as reflectivity upon the ambient glucose concentration, which allows for in situ measurements in the eye. We demonstrate that estimation errors below $2 \%$ at physiological concentrations are possible using simulations of the optical properties of the metamaterial in combination with an appropriate electrical circuitry and signal processing scheme. In the future, functionalization of our sensor with hydrogel will allow for a glucose-specific detection which is insensitive to other tear liquid substances providing both excellent selectivity and sensitivity.
\end{abstract}

\section{Introduction}

Diabetes is the direct cause of over 1.1 million deaths in 2005 , and the diabetes death rate is estimated to double by 2030. The World Health Organization (WHO) indicates in [1] that nowadays more than 220 million people have to live with diabetes. In order to allow the patients to maintain a healthy life avoiding coronary artery, peripheral arterial and cerebral vascular disease, or heart failure, early diagnosis and continuous management are crucial. Current practice for diabetes management relies on intensive insulin therapy involving frequent blood glucose measurements. Using invasive glucose sensors means that patients have to prick their finger for a drop of blood multiple times a day, about 1800 times per year, which also involves higher risk of infection. For these reasons, in the last decades new techniques have been employed to develop noninvasive devices for blood glucose monitoring.

The technologies under consideration include infrared (IR) spectroscopy [2], fluorescence spectroscopy, Raman spectroscopy, optical polarization rotation measurements, photoacoustic probes, and surface plasmon resonances.
However, none of these devices has been made commercially available or was approved to substitute direct invasive glucose measurement. In order to overcome these shortcomings, alternative approaches have been developed to measure glucose concentration in an accessible body fluid, including urine, saliva, and tear fluid.

The undeniable advantage of estimating blood glucose levels through tear fluid lies in the facts that tears are more simply and noninvasively accessible than other body fluids, more continuously obtainable, and less susceptible to dilution than urine. Tear fluid provides a unique opportunity to develop a noninvasive interface between a sensor and the body that could be used to monitor several physiological and metabolic indicators, most notably glucose. The noninvasive feature would be the main advantage of this sensing scheme.

1.1. Ophthalmic Glucose Sensing. Tear fluid is the aqueous layer on the ocular surface and has many functions as part of the optical system, that is, lubrication and nourishing. Tear fluid consists of over 20 components, including salt water, proteins, lactate, urea, pyruvate, ascorbate, glucose, as well as 
some small metallic ions. Its average rate of production lies in the range of $0.52-2.2 \mu \mathrm{L} / \mathrm{min}$; about $0.72-3.2 \mathrm{~mL}$ of tears are secreted per day.

The idea of using tear fluid as a medium for glucose monitoring has been discussed since the 1930s involving human and animal models to estimate correlation between tear glucose and blood glucose. The current technique is to collect tear fluid samples in capillary tubes and then assay the samples for glucose ex situ using standard laboratory instrumentation. Using this technique, there are many reports demonstrating that tear glucose is higher in diabetic subjects than in healthy ones and that there effectively exists correlation of tear glucose and blood glucose. It should be noted that the discrepancy of the correlation coefficient between blood glucose and tear glucose can be attributed to the different tear collection methods, for example, filter paper or microcapillary methods. In [3] a profound review of several studies resumes the most important findings.

However even after 70 years of research, there are no clinical studies that have satisfactorily resolved the relationship between tear and blood glucose concentrations. Disagreements between reports may not invalidate the correlation between tear and blood glucose because, regardless of the exact mechanism of glucose transport into tear fluid, the individual accuracy holds true for each set of experimental conditions.

An alternative approach developed recently uses an in vivo glucose sensing method that can be placed in the tear canal and that therefore reduces variability due to probe extraction technique [4]. It allows measurements to be carried out in situ. This amperometric sensor is comprised of three electrodes that are screen-printed on a flexible polyamide substrate which allows the sensor to be wound into a tight roll that fits in the tear canal for in situ monitoring.

Definitely, integrating a glucose sensor into a contact lens would provide a way to continuously and reliably sense metabolites and especially glucose in tear fluid. Different ideas to implement such a sensor have been proposed and are at present in different stages of development. They rely on placing a photonic sensor in a contact lens and envision a handheld readout unit for measuring the signal. Thus far, holographic hydrogels and fluorescent indicators have been explored as glucose-responsive elements. In [5] a polarimetric glucose sensor for monitoring ocular glucose is developed. There it is indicated that the time lag between blood glucose and anterior aqueous humor glucose concentrations was on average about five minutes. Another approach is based on a contact-lens-based sensor $[6,7]$.

It is likely that contact-lens-based glucose sensors have great potential to realize continuous and noninvasive diabetes control, that is, contact lenses have applications beyond vision correction. Luminescent/fluorescent contact-lens-based sensors represent a feasible technique because they require no electrodes or electric circuits. Further efforts are needed to improve the resolution and sensitivity of the new device and to determine a physiologically relevant and baseline tear glucose concentration $[8,9]$.

Existing methods of fluorescent glucose sensing apply Fluorescence Resonance Energy Transfer (FRET) [10]. This method is based on the dual measure, that is, the FRET and fluorescence intensity measurements. FRET is an inexpensive and very sensitive method to apply to molecule imaging. However, barriers to secure a feasible contact lens sensor include the photobleaching of fluorescence molecules, low concentration of tear samples, low fluorescence intensity, and vision influence. In addition, one safety concern is that some harming substances may be released from the lens into the body.

\subsection{Our Concept: Metamaterial-Based Biosensing. In the} present contribution a revolutionary concept for tear glucose measurement is developed. This sensing is based on the use of metamaterials, that is, artificial materials with special electromagnetic properties that do not occur naturally. In [11] a method how to manufacture such metamaterials is reported for the first time: a periodic structure design with unit cells much smaller than the wavelength of the incident radiation leads to a specific electromagnetic response on a wide spectral range. Also based on this work, the concepts of perfect lens as well as cloaking are developed in $[12,13]$. Tailoring of optical properties using the plasmonic analogue of EIT offers the possibility to obtain sharp resonances in the transmittance profile of a material leading to enhanced spectral features that can eventually be pushed to the limit of detecting single molecules [14]. Other designs such as plasmonic oligomers are also possible $[15,16]$. They rely on the formation of suitable sharp spectral Fano resonances [17].

Metamaterials are able to detect even minute changes in the dielectric properties of their environment, hence selectivity to a particular type of molecule has to be added. This is achieved by covering the metamaterial with a glucose-sensitive hydrogel [18]. When using inverse opal photonic crystals, the optical diffraction changes upon glucose exposure [19]. In Figure 1 a schematic of our proposed design is shown: a contact lens material supports a few nanometers of a gold-based metamaterial which is functionalized with glucose-sensitive hydrogel. This design is transparent in the visible and near-infrared range and thus can be designed as contact lens to be inserted into the patient's eye. The readout is carried out by an external light-emitting diode (LED) in the infrared (eye-safe range at wavelengths longer than $1.4 \mu \mathrm{m}$ ) which is used as light source, and the reflected light is captured by a photodiode whose intensity response is evaluated. Signal postprocessing stages based on regression methods allow the reliable estimation of the tear glucose content.

This new method has the potential to be extremely successful for noninvasive glucose sensing for several reasons.

(1) Glucose selectivity: this sensor does not take advantage of the rather poor optical differences between the glucose molecule and other substances contained in the surrounding fluid (blood stream, tear fluid, etc.), but rather on the ability of the glucose to change selectively the refractive index of a specific material, that is, the hydrogel in the vicinity of the metamaterial. 


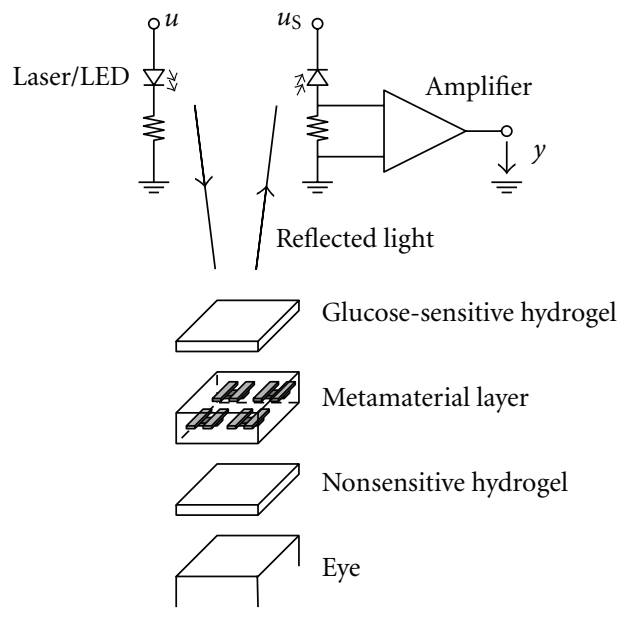

Figure 1: Schematic of the proposed design.

(2) Sensitivity: due to the fact that the metamaterial is sensitive to even minute changes in the refraction index (molecular changes in the hydrogel) the measurement can be performed in the range of physiological glucose concentration in the tear fluid.

(3) Biocompatibility: the metamaterial is made of a several nanometers thick gold structure, transparent for the human eye, and absolutely biocompatible due to the properties of noble metals. The hydrogel is commonly used for contact lenses and therefore well characterized. The optical readout is based on an eye-safe LED.

(4) Nondegrading: during the lifetime of the sensor (up to 24 hours) both the metamaterial and the hydrogel maintain its optical properties even when immersed in body fluid.

\section{Methods}

2.1. Metamaterials. The metamaterial structures are fabricated by electron beam lithography. For laboratory experiments, a $30-40 \mathrm{~nm}$ layer of gold is deposited on a $10 \times$ $10 \mathrm{~mm}^{2}$ quartz substrate using electron-beam evaporation. Next, a negative photo resist is spin-coated on top of the substrate, allowing the desired structures to be defined by electron-beam lithography. After development of the resist, directed argon ion beam etching is carried out to transfer the structure into the gold layer.

Multilayer designs can be achieved by combining this process with a stacking technique [20]. In this case, one starts with the evaporation of several gold alignment marks with a thickness of about $250 \mathrm{~nm}$ using positive resist with subsequent gold evaporation and lift-off. The first layer can then be manufactured following exactly the procedure given for a single layer. Afterwards, a spacer layer is applied by spin coating. The spacer currently consists of a hardenable photopolymer and can vary in height from ten to several hundreds

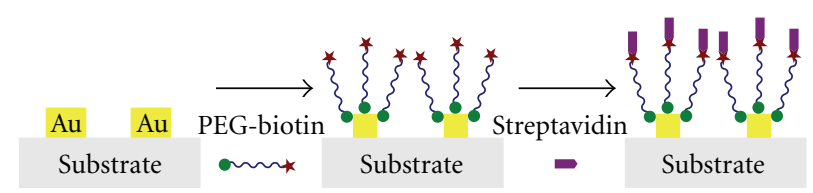

FIgURE 2: The principle of biological sensing with metamaterial gold structures.

of nanometers. Additional layers may be added by repetition of those steps while accurate alignment between the layers is assured using the gold marks during the electron beam exposure.

2.2. Biosensing. In general, broadband electromagnetic radiation in the optical domain is used to investigate the respective properties of nanostructures in sensing applications. One possibility is the recording of transmittance or reflectance spectra which exhibit characteristic dips and peaks. Due to the localized electric field in and around the metallic pattern, the resonance positions are highly sensitive to changes of the electric permittivity or the refractive index, respectively, in the nearest vicinity of the plasmonic nanostructures. Exploiting this fact allows to monitor, for example, the concentration of pure solutions on top of the structure by evaluating the shift of a distinct spectral feature [19].

However, such gold structures are not able to detect specific substances in an unfunctionalized fashion. To realize a chemically selective sensor, we have to assure that the changes in the refractive index are exclusively caused by the desired analyte. For biological sensing, the existence of molecule pairs with strong affinity can be beneficial. Ranking among the strongest noncovalent interactions known in nature, the biotin-streptavidin complex, for example, is a commonly used system for proof of concept experiments (see Figure 2). The vitamin biotin can be functionalized with a thiol group by utilising polyethylene glycol as a spacer. This allows the whole molecule to bind to the gold nanostructures. If the structure is now rinsed with an analyte containing streptavidin, the molecules will attach to the biotin and due to their presence affect the dielectric environment of the gold structure. This effect, and therefore the detectable change in the optical spectrum, will remain even after washing away other substances that may have an impact on the measurement [21].

From a conceptual point of view, the method of embedding the functionalization into a hydrogel is similar. Hydrogels are polymer networks that, due to their hydrophilic properties, absorb a considerable amount of water which causes substantial swelling. Lee et al. have shown that replacing several sites in the polymer chains with a molecule which will form a charged complex with a glucose molecule establishes a relation between the swelling of the hydrogel and the glucose concentration in the surrounding water [18]. As those changes in volume also imply a varying refractive index, they again are subject to detection by the metamaterial structure. 
The resulting spectra in both cases can be analyzed in different ways. An important value is the so-called sensitivity

$$
s_{\lambda}=\frac{\Delta \lambda}{\Delta n} \quad \text { or } \quad s_{E}=\frac{\Delta E}{\Delta n}
$$

which describes the shift in $\mathrm{nm}$ or $\mathrm{eV}$ of the resonance per refractive index unit (RIU). According to Sherry et al. the linewidth of the resonance also plays an important role [22]. Therefore one can define a figure of merit

$$
\mathrm{FOM}_{\lambda, E}=\frac{s_{\lambda, E}}{\mathrm{FWHM}},
$$

where FWHM denotes the full width at half maximum.

These values have in common that a spectrometer is needed to determine both. This rather complex and cost-extensive method is only applicable in scientific research. In commercial products it is more likely that intensity changes at a specific wavelength are evaluated. This leads to the intensity dependent sensitivity

$$
s_{I}=\frac{\Delta I}{\Delta n}
$$

and the related figure of merit

$$
\mathrm{FOM}_{I}=\frac{s_{I}}{I}
$$

describing the relative intensity change per refractive index unit.

2.3. Simulation Model. Scattering matrix theory was used to simulate the spectra of the metamaterial structures. This method which uses a Fourier modal decomposition of the electric and magnetic fields has been introduced by Whittaker and Culshaw [23] and has later been extended and improved by Tikhodeev et al. [24] as well as recently by Weiss et al. [25].

The dielectric functions for the materials used to define the periodically repeated unit cell can be retrieved from a database or entered as parameters for the Drude model.

In the definition of the structure as well as in the calculations, the design is separated into single layers, beginning at the superstrate, down to the substrate, each homogeneous along the $z$-axis. The first step is to solve Maxwell's equations for every layer.

The structured slab couples the incident light with frequency $\omega$ and wave vector $\vec{k}$ to all Bragg-orders retrieved from Maxwell's equations with the same frequency and wave vector

$$
k_{x, \vec{G}}=k_{x}+G_{x}, \quad k_{y, \vec{G}}=k_{y}+G_{y},
$$

with the reciprocal lattice vector

$$
\begin{gathered}
\vec{G}=\left(G_{x}, G_{y}, 0\right)=\frac{2 \pi}{d_{x}}\left(g_{x}, g_{y}, 0\right), \\
g_{x, y}=0, \pm 1, \pm 2, \ldots, \pm \infty
\end{gathered}
$$

and the lattice constant $d_{x}$.
Hence, the S-matrix method is able to calculate the outbound $4 N_{g}$ harmonics from the system $\left(N_{g}=2 g+1\right)$. The method is exact for $G \rightarrow \infty$. In reality, only a limited number of lattice vectors are used for the calculation. Because of the fact that the calculation time increases with $N_{g}^{3}$, computing power is the limiting factor. A typical number for $N_{g}$ is $25 \times$ 25.

The method can be accelerated and improved in accuracy by using adaptive spatial resolution and the customisation of the coordinate system, depending on the individual structure.

In the next step, the amplitudes of the waves in the single layers have to be concatenated. Therefore, the respective solutions of Maxwell's equations have to be separated into a set of eigenvectors parallel to the $z$-axis. The amplitudes of the plane waves can now be written as vectors

$$
\vec{A}(z)=\left(\begin{array}{c}
\vec{A}^{+}(z) \\
\vec{A}^{-}(z)
\end{array}\right) .
$$

All components heading to the positive (negative) $z$-direction are labelled with $+(-)$. With the aid of a so-called transfer matrix, those vectors are linked at different positions $(z$ values) in the layer:

$$
\vec{A}(z+L)=T_{L} \vec{A}(z) .
$$

The transition from one layer $(a)$ to another $(b)$ can be described similarly:

$$
\left.\vec{A}\right|_{z=z_{b, a}+0}=\left.T_{b, a} \vec{A}\right|_{z=z_{b, a}-0} .
$$

In general, it would be possible to calculate the propagation of light in layered structures using the transfer matrix formalism. However in case of evolving evanescent waves, this method may fail. This is the reason for using the scattering matrix algorithm. All amplitudes of waves incident on the sample, as well as the outbound waves, are combined into one vector:

$$
\vec{B}_{\text {in }}=\left(\begin{array}{c}
\vec{A}_{v}^{+}(z) \\
\overrightarrow{A_{s}^{-}}(z)
\end{array}\right), \quad \vec{B}_{\text {out }}=\left(\begin{array}{c}
\vec{A}_{s}^{+}(z) \\
\vec{A}_{v}^{-}(z)
\end{array}\right) .
$$

Here, $v$ means "vacuum" (above the sample), and $s$ means "substrate" (below the sample). The scattering matrix concatenates both vectors:

$$
\vec{B}_{\text {out }}=S_{v, s} \vec{B}_{\text {in }} .
$$

The whole S-matrix can be obtained by iteration, beginning with the unit matrix for $N=0$ layers and subsequently calculating the matrix for $N+1$ layers with the aid of the inverse transfer matrix.

Using scattering matrix theory, it is possible to calculate reflectance, transmittance, extinction, and absorption spectra of metallic structures. Additionally, information about the electric and magnetic field distribution can be obtained. 
2.4. Regression Methods. The aim in regression is to find a functional connection between some input $x$ (for example, $\left.x \in \mathbb{R}^{n}\right)$ in input space and some output $y \in \mathbb{R}$, that is, $y=f(x)$. Once this connection is established using some training data, it is validated by applying the regression model on data that was not used during the training process. The validated model is then employed on unknown input data for the task of output prediction. In this paper, the method of support vector machine regression (SVR) [26] is used.

Support vector machines emerged from the field of learning theory. They are constructed using training data. Compared to other learning methods, overfitting is avoided by implementing the paradigm of structural risk minimization (SRM) [27].

The first step consists of defining Vapnik's $\epsilon$-insensitive loss function

$$
e\left(x_{i}, y_{i}, f\right)=\max \left\{0,\left|y_{i}-f\left(x_{i}\right)\right|-\epsilon\right\},
$$

where $\left(x_{i}, y_{i}\right)$ is the $i$ th pair of training data. This can be thought of as a punishment for the deviation of the estimated value $f\left(x_{i}\right)$ from the given value $y_{i}$. The affine ansatz

$$
f(x)=\langle w, x\rangle+b
$$

is made, where $\langle\cdot, \cdot\rangle$ denotes the scalar product. Implementing the SRM requires minimization of the weighted sum of the capacity $(1 / 2)\langle w, w\rangle$ of the machine and the training error $\sum_{i=1}^{N} e\left(x_{i}, y_{i}, f\right)$, thus leading to

$$
\min \frac{1}{2}\langle w, w\rangle+C \sum_{i=1}^{N} e\left(x_{i}, y_{i}, f\right)
$$

Slack variables $\xi_{i}^{(*)}\left(\xi_{i}\right.$ and $\left.\xi_{i}^{*}\right)$ are introduced to account for outliers [26]. This leads to the Lagrangian function

$$
\begin{aligned}
L_{\mathrm{P}}\left(w, b, \alpha^{(*)}, \beta^{(*)}\right) & \\
= & \frac{1}{2}\langle w, w\rangle+C \sum_{i=1}^{N}\left(\xi_{i}+\xi_{i}^{*}\right)-\sum_{i=1}^{N}\left(\beta_{i} \xi_{i}+\beta_{i}^{*} \xi_{i}^{*}\right) \\
& -\sum_{i=1}^{N} \alpha_{i}\left(\left\langle w, x_{i}\right\rangle+b-y_{i}+\epsilon+\xi_{i}\right) \\
& -\sum_{i=1}^{N} \alpha_{i}^{*}\left(y_{i}-\left\langle w, x_{i}\right\rangle-b+\epsilon+\xi_{i}^{*}\right)
\end{aligned}
$$

in primal space, which has to be minimized with respect to the primal variables $w$ and $b$ and maximized with respect to the dual variables $\alpha^{(*)}$ and $\beta^{(*)}$, which are the Lagrange multipliers.

Plugging in the necessary conditions for a saddle point yields the Lagrangian function

$$
\begin{aligned}
L_{\mathrm{D}}\left(\alpha^{(*)}\right)= & -\frac{1}{2} \sum_{i, j=1}^{N}\left(\alpha_{i}-\alpha_{i}^{*}\right)\left(\alpha_{j}-\alpha_{j}^{*}\right)\left\langle x_{i}, x_{j}\right\rangle \\
& -\sum_{i=1}^{N}\left(\epsilon-y_{i}\right) \alpha_{i}-\sum_{i=1}^{N}\left(\epsilon+y_{i}\right) \alpha_{i}^{*}
\end{aligned}
$$

in dual space, which has to be maximized with respect to $\bar{\alpha}=$ $\left(\alpha, \alpha^{*}\right)$ subject to the constraints

$$
\begin{gathered}
\sum_{i=1}^{N}\left(\alpha_{i}-\alpha_{i}^{*}\right)=0, \\
0 \leq \alpha_{i} \leq C, \\
0 \leq \alpha_{i}^{*} \leq C .
\end{gathered}
$$

In order to achieve nonlinear regression, a mapping

$$
x \in \mathbb{R}^{n} \longmapsto \Phi(x) \in \mathbb{R}^{f}
$$

from input space to feature space is introduced. Usually $f \gg n$ holds true. The nonlinear regression in input space corresponds to a linear regression in some feature space. Instead of actually performing the mapping, which might be computationally expensive, the so-called kernel trick is applied. It depends on the fact that the training data only occurs in the form of scalar products and that scalar products in feature space can be calculated in input space using the kernel $k(\cdot, \cdot)$ according to

$$
k\left(x_{i}, x_{j}\right)=\left\langle\Phi\left(x_{i}\right), \Phi\left(x_{j}\right)\right\rangle .
$$

\section{Results}

3.1. Simulated Reflectance Spectra. For a first overview, we simulated spectra for a broad concentration range of aqueous glucose solutions on top of different metamaterials, namely, a simple plasmonic dipole and a stacked EIT-type metamaterial. Starting with pure water, we added

$$
20 \cdot 0.5^{(10-n)}, \quad n=1,2,3, \ldots, 10,
$$

weight percent of glucose, corresponding to values from about $40 \mathrm{mg} / \mathrm{dL}$ up to $22 \mathrm{~g} / \mathrm{dL}$.

Our EIT metamaterial uses a $60 \mathrm{~nm}$ displacement of the dipole bar from the central symmetry axis. The length of the dipole bar is $340 \mathrm{~nm}$, whereas the quadrupole bar is $345 \mathrm{~nm}$ long. Their width is $80 \mathrm{~nm}$, the gold thickness is $40 \mathrm{~nm}$, and the spacer thickness is $70 \mathrm{~nm}$.

The simple dipole structure shows one distinct peak, whereas the coupled dipole and quadrupole antenna develops an additional reflectance dip in the center of the broad peak (Figures 3(a)-3(d)). Highly confined electric fields are responsible for sensitivity and the possibility of extremely small sensing volumes (Figure 3(e)). The resulting sensitivities are $s_{I}=4.5 / \mathrm{RIU}$ and $s_{\lambda}=625 \mathrm{~nm} / \mathrm{RIU}$. The FOM $\mathrm{F}_{\lambda}$ is 6.0 , and the $\mathrm{FOM}_{I}$ is 9.5 .

3.2. Sensitivity Analysis. This section deals with the identification of those parameters and noise contributions that may have influence on the expected measurement results. To this end, the metamaterial simulation tool is extended by a model of the signal processing units containing noise sources and nonstationary parameter sets. The block diagram used for the simulation is depicted in Figure 4. The source consists of a modulated steering signal $\tilde{u}(t)$ that drives the laser diode, 


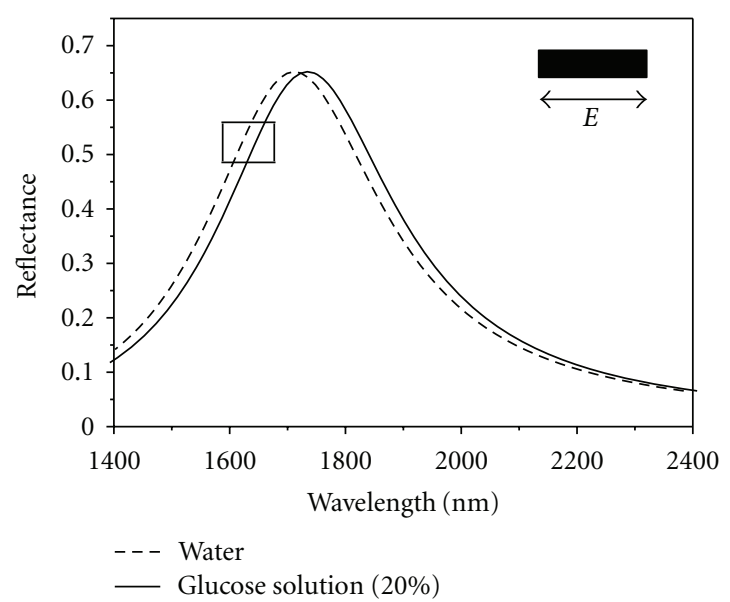

(a)

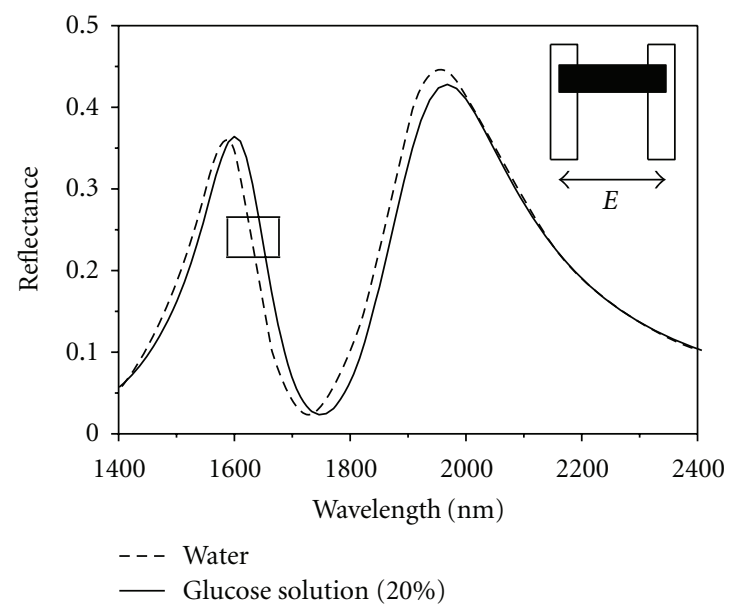

(c)

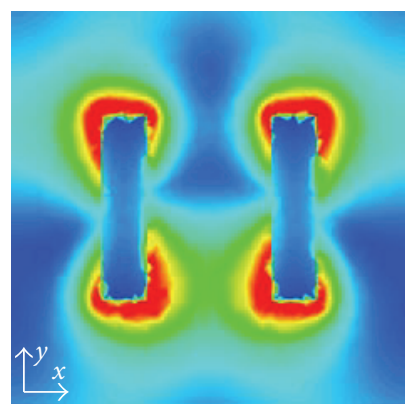

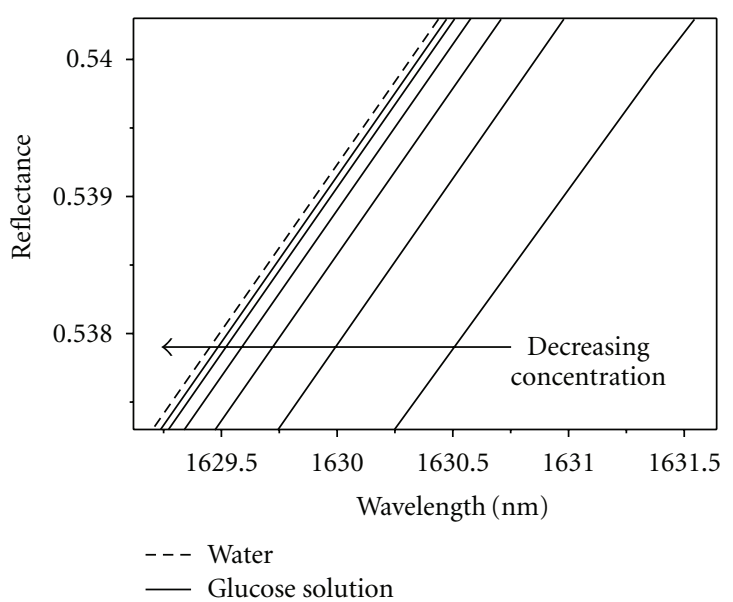

(b)

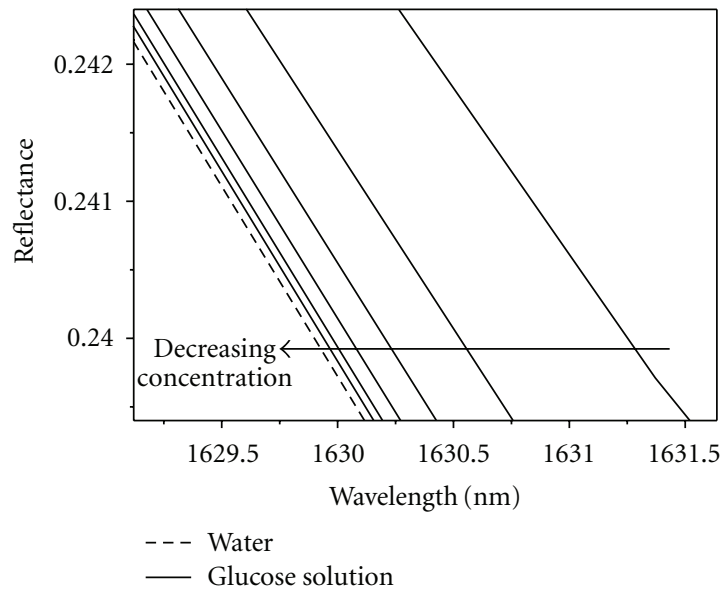

(d)

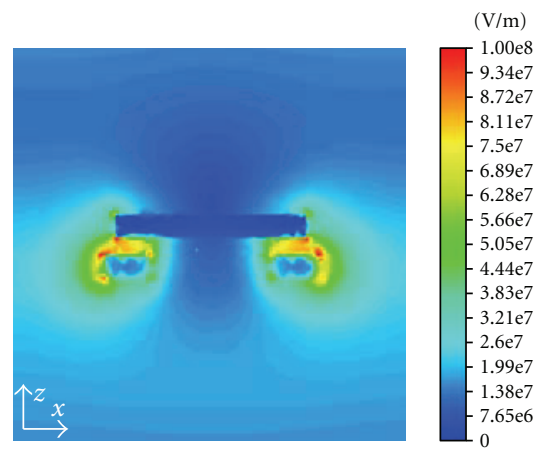

(e)

FIgURE 3: S-matrix simulations of a simple dipole structure (a) and a coupled dipole and quadrupole antenna (c) with water and 20\% glucose solution on top. A detailed view for smaller concentrations ranging from $1.25 \%$ down to $0.04 \%$ is given in (b) and (d), respectively. Figure (e) depicts the time averaged absolute values of the electric field in and around the structure at the resonance position.

from which both the output power $\widetilde{P}_{0}(t)$ and the actual wavelength $\lambda(t)$ are measured. Laser diodes show some deviation $\Delta \lambda$ from their nominal wavelength due to the manufacturing processes, and their wavelength also varies significantly with temperature.

The contact lens block contains the embedded metamaterial spectra which functionally attenuates the power output
$\widetilde{P}_{0}(t)$ to the received reflected power $\widetilde{P}(t)$, depending on the actual wavelength $\lambda(t)$.

The reflected laser ray is detected by the photodiode. Figure 5 shows a scheme of the circuitry which is used to amplify the current of the photodiode [28]. A characteristic feature for this kind of feedback amplifier is the virtual ground at the node of the inverting input terminal which 


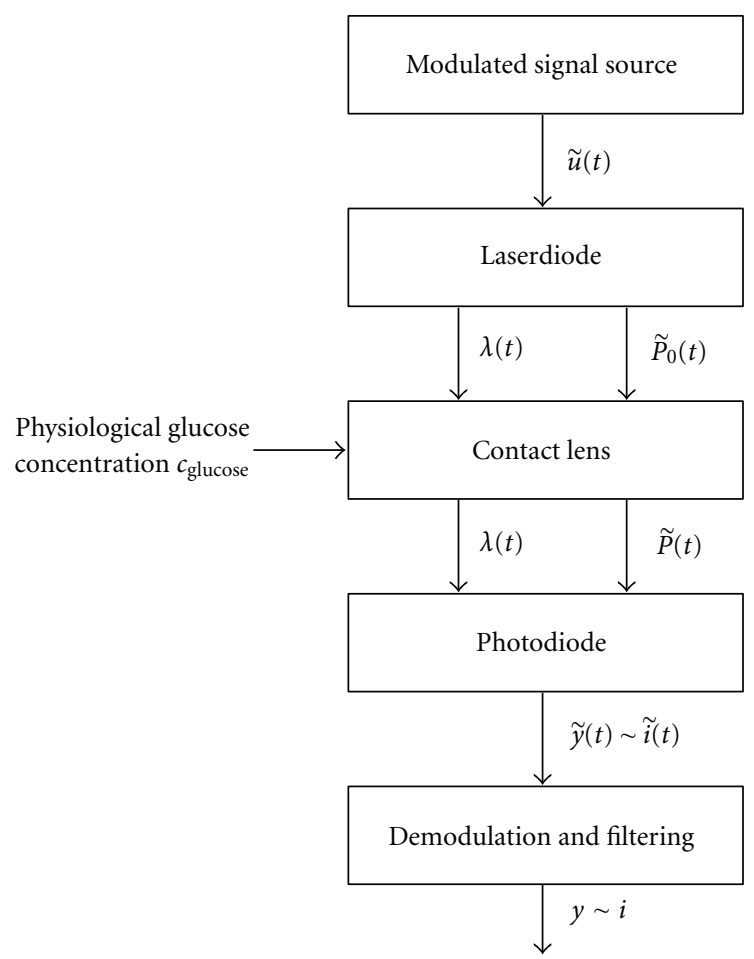

FIGURE 4: Block diagram used for simulation including signal processing scheme and embedded metamaterial model.

enables a higher bandwidth [29]. The bias resistor $R_{\mathrm{B}}$ reduces the effect of the bias current, which is nonzero for any real operational amplifier. In the simulation model, the current $\widetilde{i}(t)$ of the photodiode is converted to an output voltage $\tilde{y}(t)$ using unit amplification.

Finally, demodulation and filtering are performed. In order to avoid higher frequency noise contributions and to detect the steady state value, low-pass filtering is performed and its output signal $y$ is then evaluated.

The selected wavelengths represent the spectra at their steepest slope. In fact, for the dipole metamaterials, $\lambda_{\text {dipole }}=$ $1823 \mathrm{~nm}$ is used, and for the EIT metamaterials, $\lambda_{\mathrm{EIT}}=$ $1631 \mathrm{~nm}$.

3.2.1. Noise Sources. Considering this simulation model the noise sources are analyzed qualitatively in order to find out which of them are relevant.

According to $[28,30]$, the total noise in a photodiode is the sum of its thermal noise (Johnson-Nyquist noise), shot noise, $1 / \mathrm{f}$ noise, and generation-recombination noise.

The thermal noise $I_{\mathrm{th}}^{2}$ and the shot noise $I_{\mathrm{sh}}^{2}$ are calculated according to

$$
\begin{aligned}
& I_{\mathrm{th}}^{2}=\frac{4 k T \Delta f}{R}, \\
& I_{\mathrm{sh}}^{2}=2 q I_{\mathrm{D}} \Delta f,
\end{aligned}
$$

with the real part $R$ of the impedance, Boltzmann's constant $k=1.38 \times 10^{-23} \mathrm{~J} / \mathrm{K}$, the temperature $T$ in Kelvin, the electron charge $q=1.602 \times 10^{-19} \mathrm{C}$, the diode current $I_{\mathrm{D}}$, and

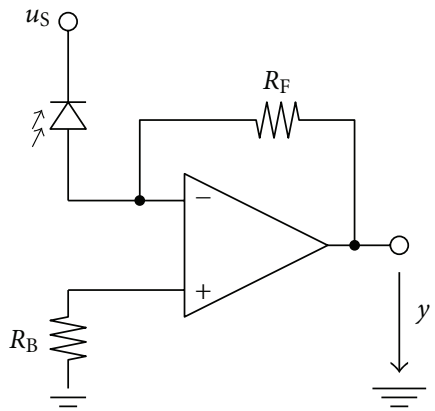

Figure 5: Circuitry used for the amplification of the photodiode current.

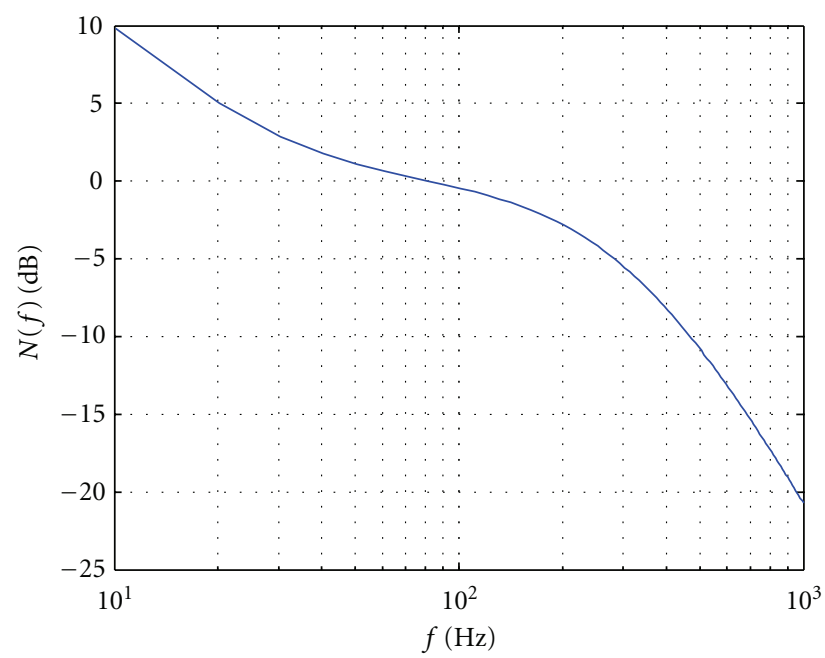

FIGURE 6: Noise filter used to shape white thermal and shot noise.

the noise bandwidth $\Delta f$. Both thermal and shot noise are modelled as white noise. They form the $0 \mathrm{~dB}$ line of the noise filter (Figure 6), which takes into account 1/f-noise and generation-recombination noise. In the presented simulations, the sampling frequency $f_{S}=2000 \mathrm{~Hz}$ is used. The Nyquist frequency is therefore $f_{N}=f_{S} / 2=1000 \mathrm{~Hz}$.

According to the modeled noise sources the noise power of the photodiode noise decreases as the frequency increases. Exactly for this reason, the laser signal is modulated with $f_{\text {mod }}$, which is chosen to be $f_{\text {mod }}=f_{N} / 2=500 \mathrm{~Hz}$ for the presented simulations, thus taking advantage of the noise reduction at higher frequencies. This fact becomes apparent when analyzing the power spectral density (PSD) of the photodiode current: almost the entire signal power is contained within the band around $f_{\text {mod }}$ (see Figure 7 ), where the noise can be disregarded. Thus we conclude that the influence of the photodiode noise can be neglected with regard to the glucose measurement results.

3.2.2. Parameter Variation. Significant parameter variation is expected with regard to temperature and laser wavelength. 


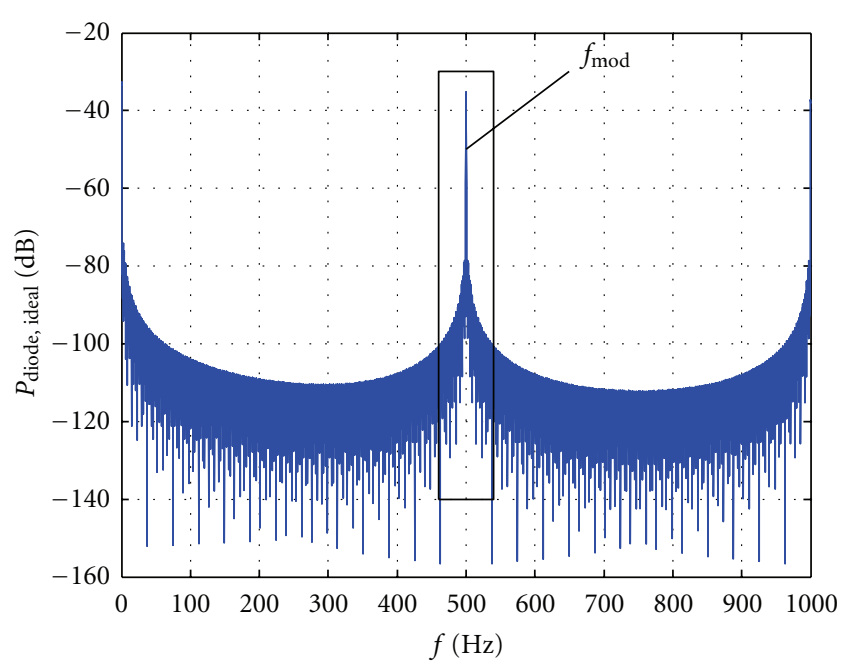

(a)

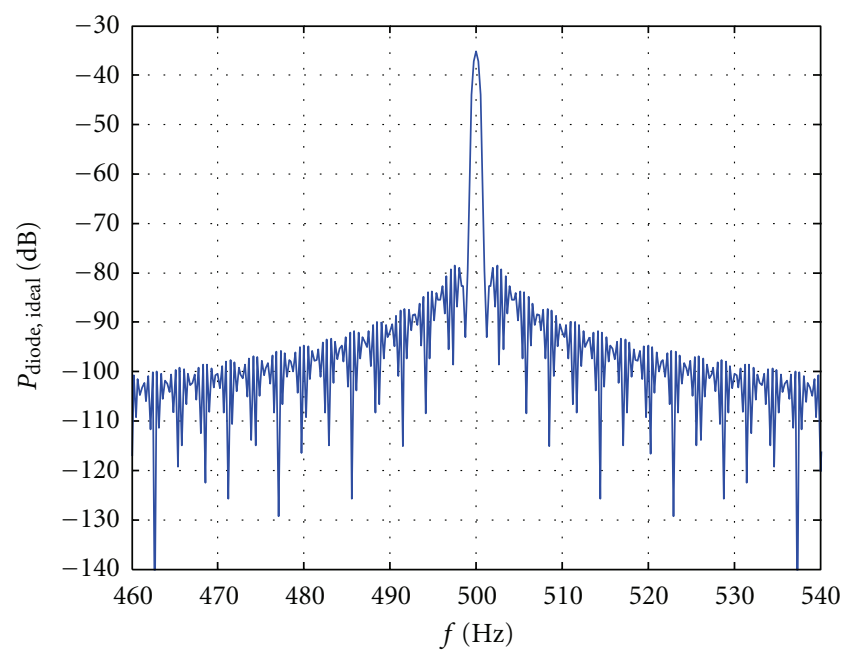

(b)

FIGURE 7: PSD of the ideal photodiode current signal. (a) depicts the entire spectrum, whereas (b) shows a detailed view of the power spectral density around the modulation frequency $f_{\bmod }$.

(i) Temperature. A change in temperature modifies the wavelength of the laser and thus is considered to be a crucial parameter.

The influence of the temperature is investigated for a constant concentration $c_{\text {glucose }}=10 \%$ and for both metamaterial structures (dipole and EIT). The results are depicted in Figure 8, where we observe that the normalized photodiode current sensitivity to temperature deviations is of $S_{T}=$ $\Delta I / \Delta T=0.017 \mathrm{~mA} / \mathrm{K}$ for dipole metamaterial and of $S_{T}=$ $0.03 \mathrm{~mA} / \mathrm{K}$ for the EIT metamaterial. Therefore, the temperature drift must be taken into account when evaluating measurements.

(ii) Wavelength. The deviation $\Delta \lambda$ of the laser wavelength from its nominal value is investigated in relation to the photodiode current. Figure 9 shows the photodiode current over $\Delta \lambda$ for $\Delta \lambda_{\max }= \pm 3 \mathrm{~nm}$ for both the dipole and the EIT

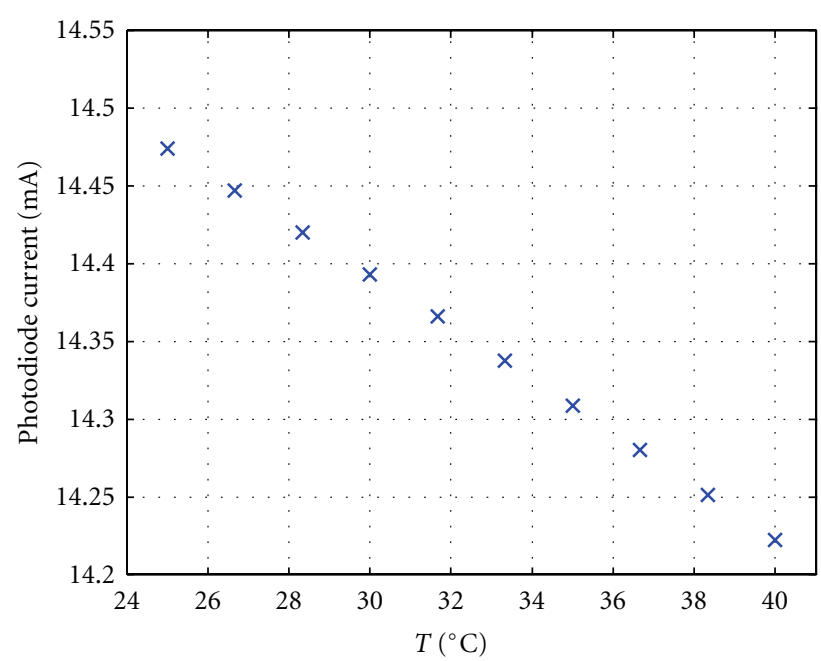

(a)

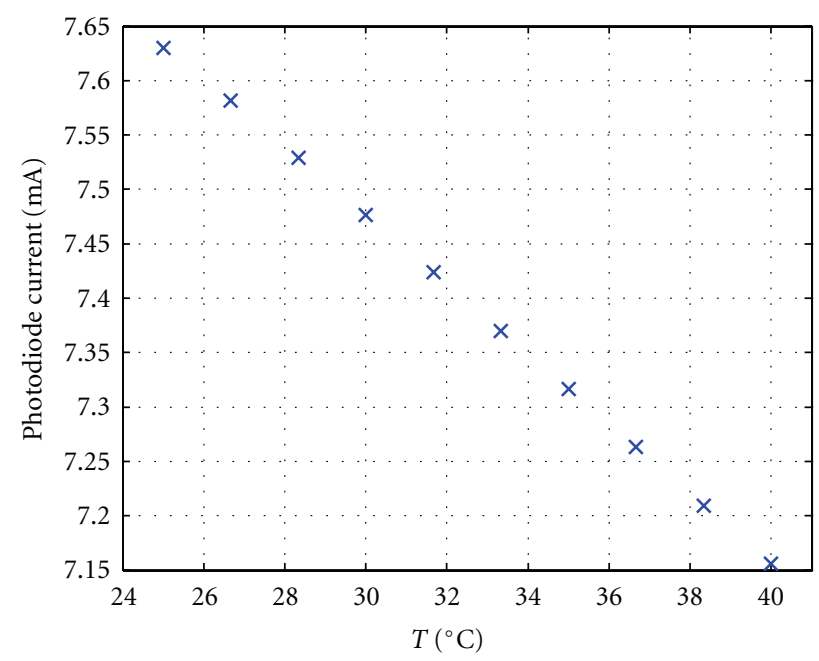

(b)

Figure 8: Photodiode current over temperature $T$ for the dipole metamaterial (a) and for the EIT metamaterial (b).

metamaterial for a constant concentration of $c_{\text {glucose }}=10 \%$. The resulting wavelength deviations sensitivity is $S_{\lambda}=$ $\Delta I / \Delta \lambda=0.05 \mathrm{~mA} / \mathrm{nm}$ for dipole metamaterial and $S_{\lambda}=$ $0.1 \mathrm{~mA} / \mathrm{nm}$ for EIT metamaterial.

Thus, the laser diode wavelength drift is an even more significant parameter than temperature.

3.2.3. Glucose Concentration Sensitivity. Finally an evaluation of the measurement sensitivity is performed. In Figure 10 , the photodiode current is shown as a function of the glucose concentration for both dipole metamaterial and EIT metamaterial. One can observe that the dynamic range of the current is larger for the EIT metamaterial due to the steeper slope. The measurement sensitivity results in $S=\Delta I / \Delta c_{\text {glucose }}=6 \times 10^{-5} \mathrm{~mA} /[\mathrm{mg} / \mathrm{dL}]$ for the dipole metamaterial and in $S=10 \times 10^{-5} \mathrm{~mA} /[\mathrm{mg} / \mathrm{dL}]$ for EIT metamaterial. 


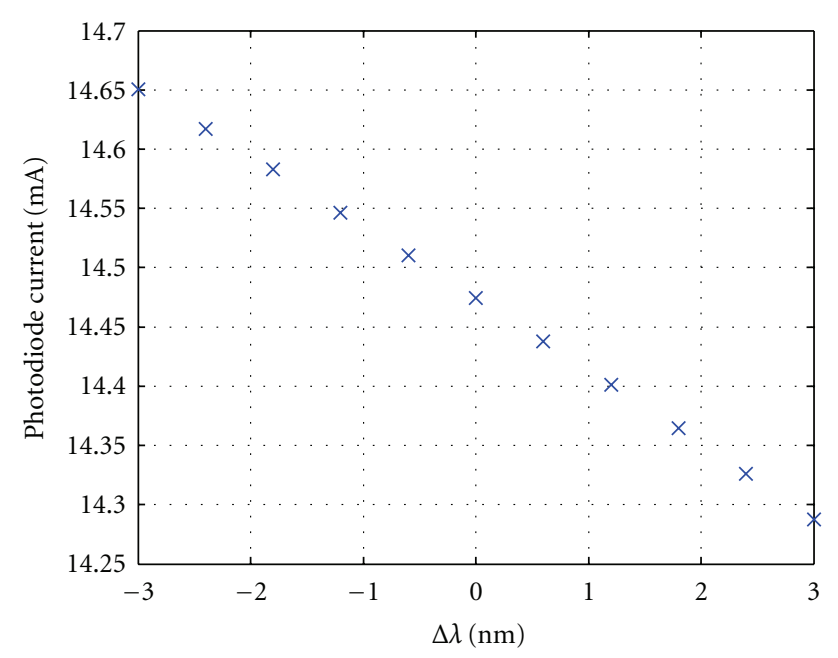

(a)

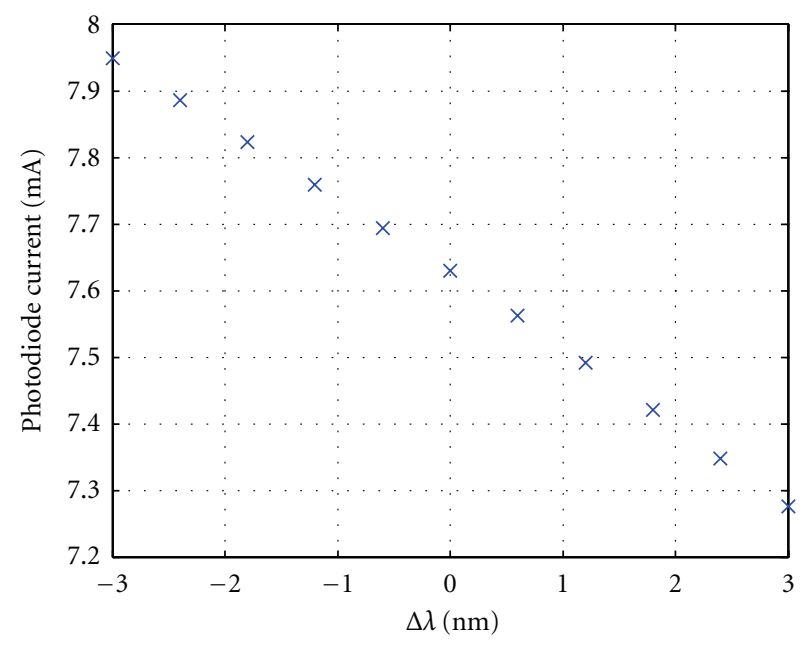

(b)

FIGURE 9: Photodiode current over wavelength deviation $\Delta \lambda$ for the dipole metamaterial (a) and for the EIT metamaterial (b).

3.3. Estimation Using Support Vector Regression. The analysis of measurement errors in glucose monitoring systems presents a particularly troublesome problem, because the importance (that is, the clinical consequence) of any particular error depends on the absolute value of both the reference and measured values and not just on the percentage of deviation. Moreover, this dependence is not easily described by any simple mathematical relationship. Although Error Grid Analysis (EGA) was introduced in the mid-1980s [31], an evaluation based on standardized signal processing and statistical tools is more meaningful for a preclinical analysis, which suits our purpose.

In the presented systems the glucose level concentration corresponds to the concentration used in the spectrum simulation. In order to obtain a predicted concentration, support vector regression is employed. The training data is the simulated photodiode current as independent variable and the associated glucose concentration as dependent variable. The results are validated using $\mathrm{k}$-fold cross-validation.

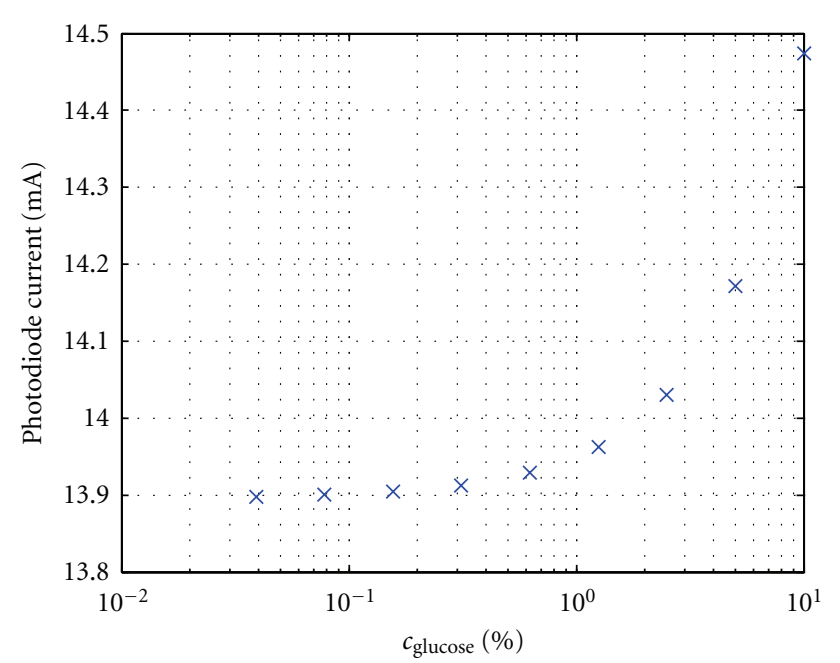

(a)

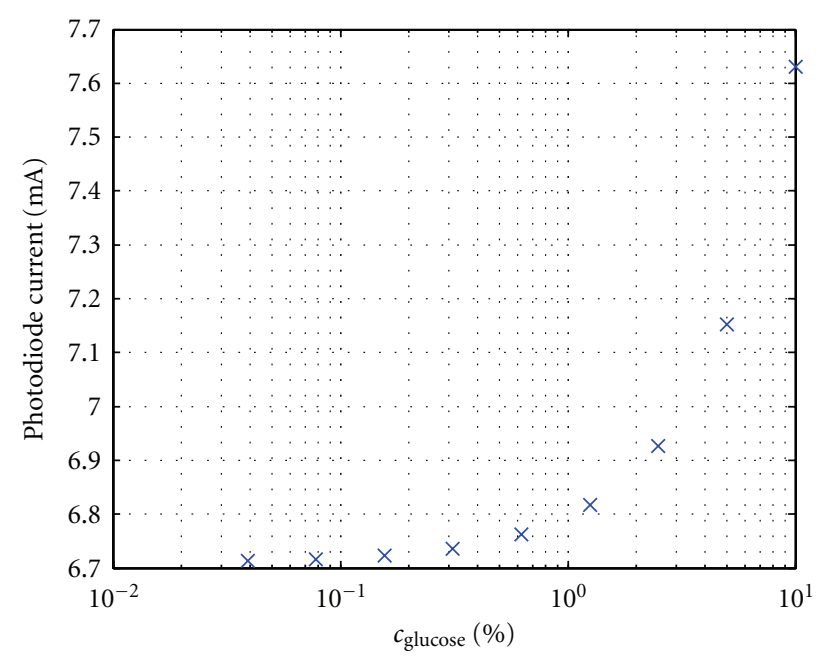

(b)

FIGURE 10: Photodiode current over glucose concentration for the dipole metamaterial (a) and for the EIT metamaterial (b).

Support vector regression will also be employed in the actual measurement device. In that case, the training data consists of the measured photodiode current as independent variable as well as the associated glucose concentration as dependent variable. Given a measured photodiode current, the SVR is used to predict the corresponding glucose level.

Two different kernels are employed for the SVR [26].

(1) Gaussian radial basis function kernel

$$
k\left(x_{i}, x_{j}\right)=\exp \left(-\frac{\left\|x_{i}-x_{j}\right\|}{2 \sigma^{2}}\right) .
$$

(2) Complete polynomial of degree $d$

$$
k\left(x_{i}, x_{j}\right)=\left(\left\langle x_{i}, x_{j}\right\rangle+1\right)^{d} .
$$




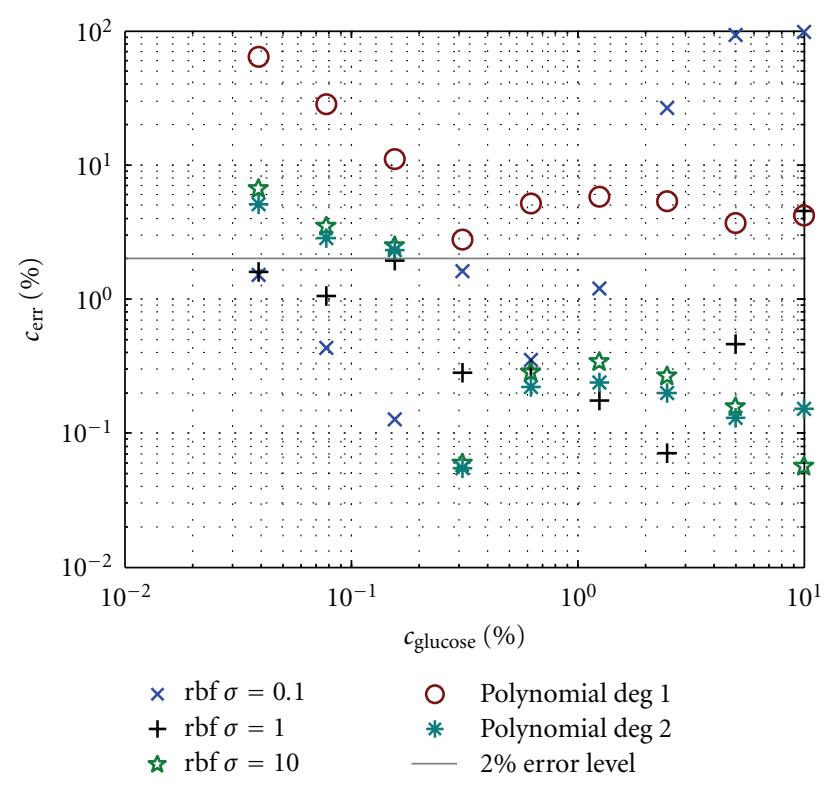

FIGURE 11: Statistical evaluation using k-fold cross-validation for nominal temperature $T=25^{\circ} \mathrm{C}$ and nominal wavelength $(\Delta \lambda=$ $0 \mathrm{~nm}$ ). The grey line denotes the $2 \%$ error level.

The first simulations are carried out for $T=25^{\circ} \mathrm{C}$ and the nominal wavelength of the respective laser; see Figure 11. The relative error

$$
c_{\mathrm{err}}=\frac{\left|c-c_{\mathrm{est}}\right|}{|c|} \cdot 100
$$

as function of the estimated glucose concentration $c_{\text {est }}$ given in percent is depicted over the respective glucose concentration. The polynomial kernel of degree $d=1$ corresponds to linear regression. It turns out that the error is very large in this case. That motivates the use of nonlinear support vector regression. The Gaussian radial basis function kernel with $\sigma=1$ yields estimation errors below $2 \%$ for physiological concentrations.

Next, the influence of temperature variation is investigated; see Figure 12. The Gaussian radial basis function kernel with $\sigma=1$ is used in each simulation run. The temperature $T$ is varied from $25^{\circ} \mathrm{C}$ to $40^{\circ} \mathrm{C}$.

Finally, simulations are carried out varying the wavelength deviation $\Delta \lambda=-1 \mathrm{~nm} \ldots 1 \mathrm{~nm}$ (Figure 13). Once again, the Gaussian radial basis function kernel with $\sigma=1$ is employed.

\section{Discussion}

4.1. Metamaterial Shape. The metamaterial shape (dipole or EIT) plays a key role for the available maximum slope in the spectrum. A steeper slope in turn leads to a broader dynamic range of the photodiode current. Therefore, the use of the EIT shape is preferable.

When comparing simple dipole plasmonic structures with plasmonic EIT sensors, we find that in terms of concentration sensitivity, the EIT concept is superior by at least a factor of two over the simple dipole. However, as a drawback,

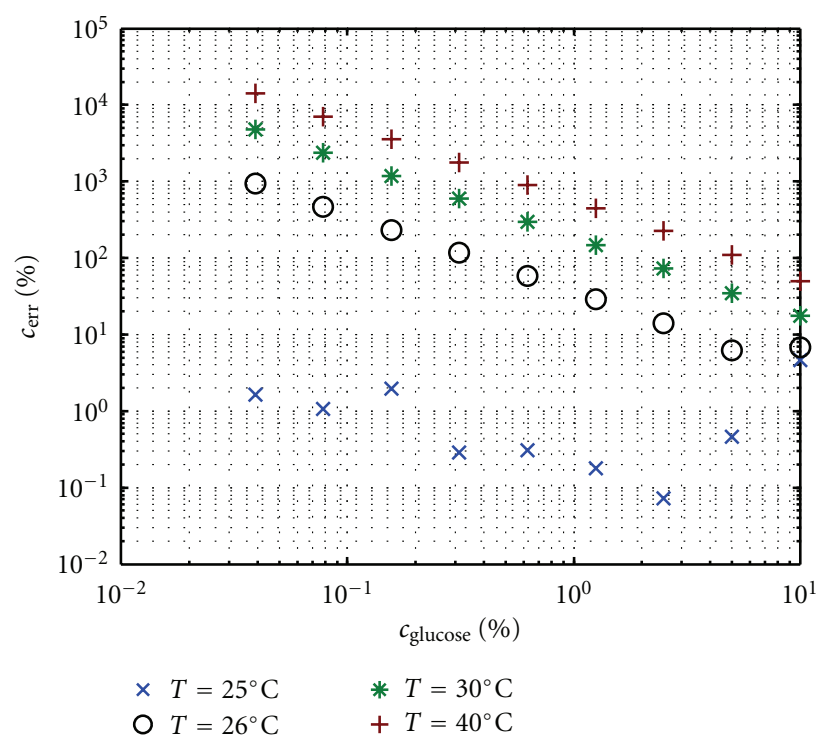

Figure 12: Statistical evaluation using k-fold cross validation for nominal wavelength $(\Delta \lambda=0 \mathrm{~nm})$, showing the effect of temperature variation.

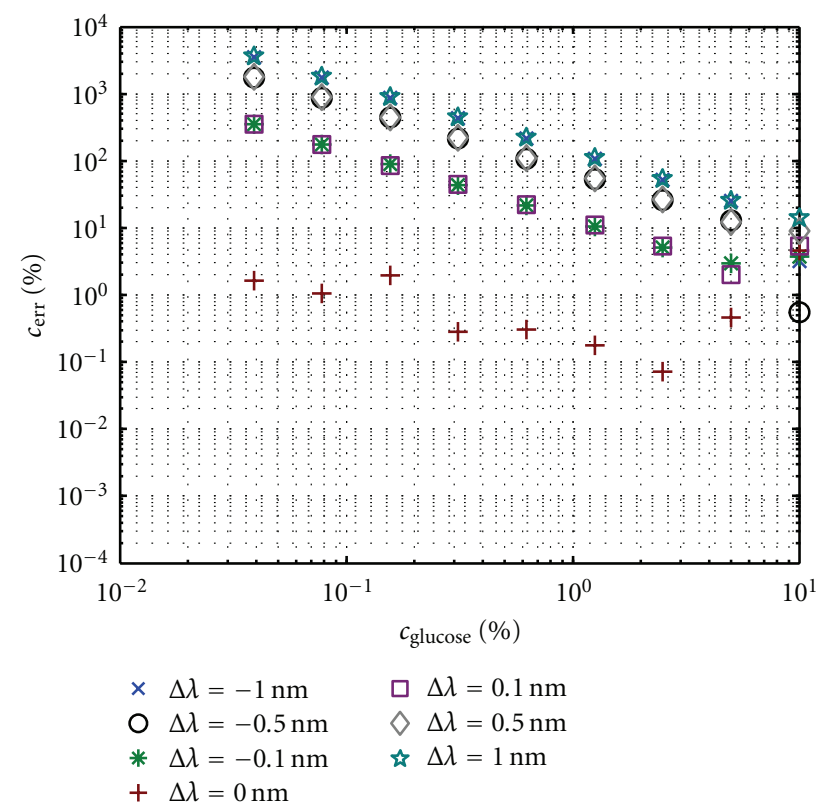

Figure 13: Statistical evaluation using k-fold cross validation for nominal temperature $T=25^{\circ} \mathrm{C}$, showing the effect of wavelength deviation.

the EIT concept due to its steeper resonances is also more prone to wavelength shifts due to temperature variations. However, this problem can be circumvented by using a temperature stabilization scheme for the laser diode.

On top of that, the EIT shape offers four specific wavelengths with a large slope, compared to the dipole shape with only two points. This increases the flexibility for choosing a specific wavelength, as not every wavelength is available in commercial lasers. 
4.2. Statistical Evaluation. Special care has to be taken when the temperature or the wavelength differ from their nominal values. As the SVR does not contain those parameters, the prediction deteriorates. The relative errors for the determination of the glucose concentration are presented in Section 3.3. They become very large even for small variations of those parameters.

In order to overcome this issue, temperature will be included as independent variable in the SVR in future work. On top of that, the system will be calibrated in order to correct the wavelength deviation of a specific laser.

\section{Conclusion and Further Work}

5.1. Proof of Concept. The present paper demonstrates a novel concept for in vivo sensing of glucose using metamaterials in combination with automatic learning systems.

The novelty of the approach lies in the fact that this sensor does not take advantage of the rather poor optical differences between the glucose molecule and other substances contained in the surrounding fluid (blood stream, tear fluid, etc.), but rather on the ability of the glucose to change selectively the refractive index of a specific metamaterial.

High sensitivity of our detection scheme is warranted because metamaterials are able to detect even minute changes in the dielectric properties of their environment. The basic concept relies on a contact lens material that supports a few nanometers of a gold-based metamaterial which is functionalized with glucose-sensitive hydrogel. This design is transparent in the visible and near-infrared range and thus can be designed as contact lens to be inserted into the patient's eye. The readout is carried out by an external LED, and the reflected light is captured by a photodiode whose intensity response is evaluated. Signal postprocessing stages based on regression methods allow the reliable estimation of the tear glucose content.

A complex simulation environment is built to evaluate the main signal contributions together with the most important noise sources as well as the most relevant parameter uncertainties. The simulation results have shown that estimation errors below $2 \%$ at physiological concentrations are possible.

5.2. Functionalization with Glucose-Sensitive Hydrogel for Contact Lens Implementation. The plasmonic sensor concept has proven to be suitable for glucose detection at physiological concentrations.

In the future, we are going to implement a glucose selective layer on the plasmonic structure. This includes a functionalization layer with a glucose-specific hydrogel $[18,32]$.

The hydrogel allows only glucose to penetrate the functionalization layer and not other chemical agents that are present in the tear fluid.

Furthermore, the hydrogel is quite biocompatible, in particular for the human eye environment. In fact, soft contact lenses already use those kinds of hydrogels as surface layers.

\section{Acknowledgments}

The authors would like to thank the Ministerium für Wissenschaft, Forschung und Kunst Baden-Württemberg as well as BMBF and DFG (Open Access Publishing Fonds) for granting financial support.

\section{References}

[1] World Health Organization, Diabetes. Fact Sheet N 312, WHO, Geneva, Switzerland, 2010, http://www.who.int/mediacentre/ factsheets/fs312/en/.

[2] Y. C. Shen, A. Davies, E. Linfield, T. Elsey, P. Taday, and D. Arnone, "The use of Fourier-transform infrared spectroscopy for the quantitative determination of glucose concentration in whole blood," Physics in Medicine and Biology, vol. 48, no. 13, pp. 2023-2032, 2003.

[3] J. Zhang, W. Hodge, C. Hutnick, and X. Wang, "Noninvasive diagnostic devices for diabetes through measuring tear glucose," Journal of Diabetes Science and Technology, vol. 5, no. 1, pp. 166-172, 2011.

[4] J. Wang, "In vivo glucose monitoring: towards 'Sense and Act' feedback-loop individualized medical systems," Talanta, vol. 75, no. 3, pp. 636-641, 2008.

[5] B. H. Malik and G. L. Coté, "Modeling the corneal birefringence of the eye toward the development of a polarimetric glucose sensor," Journal of Biomedical Optics, vol. 15, no. 3, pp. 037012-037018, 2010.

[6] C. O'Donnell, N. Efron, and A. J. M. Boulton, "A prospective study of contact lens wear in diabetes mellitus," Ophthalmic and Physiological Optics, vol. 21, no. 3, pp. 127-138, 2001.

[7] W. March, B. Long, W. Hofmann, D. Keys, and C. McKenney, "Safety of contact lenses in patients with diabetes," Diabetes Technology and Therapeutics, vol. 6, no. 1, pp. 49-52, 2004.

[8] V. L. Alexeev, S. Das, D. N. Finegold, and S. A. Asher, "Photonic crystal glucose-sensing material for noninvasive monitoring of glucose in tear fluid," Clinical Chemistry, vol. 50, no. 12, pp. 2353-2360, 2004.

[9] R. Badugu, J. R. Lakowicz, and C. D. Geddes, "Wavelengthratiometric probes for the selective detection of fluoride based on the 6-aminoquinolinium nucleus and boronic acid moiety," Journal of Fluorescence, vol. 14, no. 6, pp. 693-703, 2004.

[10] M. R. G. A. Ballerstadt, C. Evans, R. McNichols, and A. Gowda, "Concanavalin a for in vivo glucose sensing: a biotoxicity review," Biosensors and Bioelectronics, vol. 22, no. 2, pp. 275-284, 2006.

[11] J. B. Pendry, A. J. Holden, D. J. Robbins, and W. J. Stewart, "Magnetism from conductors and enhanced nonlinear phenomena," IEEE Transactions on MTT, vol. 47, no. 11, pp. 20752084, 1999.

[12] J. B. Pendry, "Negative refraction makes a perfect lens," Physical Review Letters, vol. 85, no. 18, pp. 3966-3969, 2000.

[13] J. B. Pendry, D. Schurig, and D. R. Smith, "Controlling electromagnetic fields," Science, vol. 312, no. 5781, pp. 1780-1782, 2006.

[14] N. Liu, T. Weiss, J. Kästel, M. Fleischhauer, T. Pfau, and H. Giessen, "Plasmonic analogue of electromagnetically induced transparency at the Drude damping limit," Nature Materials, vol. 8, no. 9, pp. 758-762, 2009.

[15] M. Hentschel, M. Saliba, R. Vogelgesang, H. Giessen, A. P. Alivisatos, and N. Liu, "Transition from isolated to collective modes in plasmonic oligomers," Nano Letters, vol. 10, no. 7, pp. 2721-2726, 2010. 
[16] M. Hentschel, D. Dregely, R. Vogelgesang, H. Giessen, and N. Liu, "Plasmonic oligomers: the role of individual particles in collective behavior," ACS Nano, vol. 5, no. 3, pp. 2042-2050, 2011.

[17] B. Lukyanchuk, N. I. Zheludev, S. A. Maier et al., "The Fano resonance in plasmonic nanostructures and metamaterials," $\mathrm{Na}$ ture Materials, vol. 9, no. 9, pp. 707-715, 2010.

[18] Y.-J. Lee, S. A. Pruzinsky, and P. V. Braun, "Glucose-sensitive inverse opal hydrogels: analysis of optical diffraction response," Langmuir, vol. 20, no. 8, pp. 3096-3106, 2004.

[19] N. Liu, T. Weiss, M. Mesch et al., "Planar metamaterial analogue of electromagnetically induced transparency for plasmonic sensing," Nano Letters, vol. 10, no. 4, pp. 1103-1107, 2010.

[20] N. Liu, H. Guo, L. Fu, S. Kaiser, H. Schweizer, and H. Giessen, "Realization of three-dimensional photonic metamaterials at optical frequencies,” Nature Materials, vol. 7, no. 1, pp. 31-37, 2008.

[21] G. Raschke, S. Kowarik, T. Franzl et al., "Biomolecular recognition based on single gold nanoparticle light scattering," Nano Letters, vol. 3, no. 7, pp. 935-938, 2003.

[22] L. J. Sherry, R. Jin, C. A. Mirkin, G. C. Schatz, and R. P. van Duyne, "Localized surface plasmon resonance spectroscopy of single silver triangular nanoprisms," Nano Letters, vol. 6, no. 9, pp. 2060-2065, 2006.

[23] D. M. Whittaker and I. S. Culshaw, "Scattering-matrix treatment of patterned multilayer photonic structures," Physical Review B, vol. 60, no. 4, pp. 2610-2618, 1999.

[24] S. G. Tikhodeev, A. L. Yablonskii, E. A. Muljarov, N. A. Gippius, and T. Ishihara, "Quasiguided modes and optical properties of photonic crystal slabs," Physical Review B, vol. 66, no. 4, Article ID 045102, pp. 451021-4510217, 2002.

[25] T. Weiss, G. Granet, N. A. Gippius, S. G. Tikhodeev, and H. Giessen, "Matched coordinates and adaptive spatial resolution in the Fourier modal method," Optics Express, vol. 17, no. 10, pp. 8051-8061, 2009.

[26] B. Schölkopf and A. J. Smola, Learning with Kernels: Support Vector Machines, Regularization, Optimization, and Beyond, MIT Press, Cambridge, UK, 2002.

[27] L. Wang, Support Vector Machines: Theory and Applications, Springer, New York, NY, USA, 2005.

[28] C. D. Motchenbacher and J. A. Connelly, Low-Noise Electronic System Design, John Wiley \& Sons, New York, NY, USA, 1993.

[29] U. Tietze and C. Schenk, Halbleiterschaltungstechnik, Springer, New York, NY, USA, 2002.

[30] F. N. Hooge, "1/f noise sources," IEEE Transactions on Electron Devices, vol. 41, no. 11, pp. 1926-1935, 1994.

[31] W. L. Clarke, D. Cox, L. A. Gonder-Frederick, W. Carter, and S. L. Pohl, "Evaluating clinical accuracy of systems for selfmonitoring of blood glucose," Diabetes Care, vol. 10, no. 5, pp. 622-628, 1987.

[32] S. A. Asher, V. L. Alexeev, A. V. Goponenko et al., "Photonic crystal carbohydrate sensors: low ionic strength sugar sensing," Journal of the American Chemical Society, vol. 125, no. 11, pp. 3322-3329, 2003. 

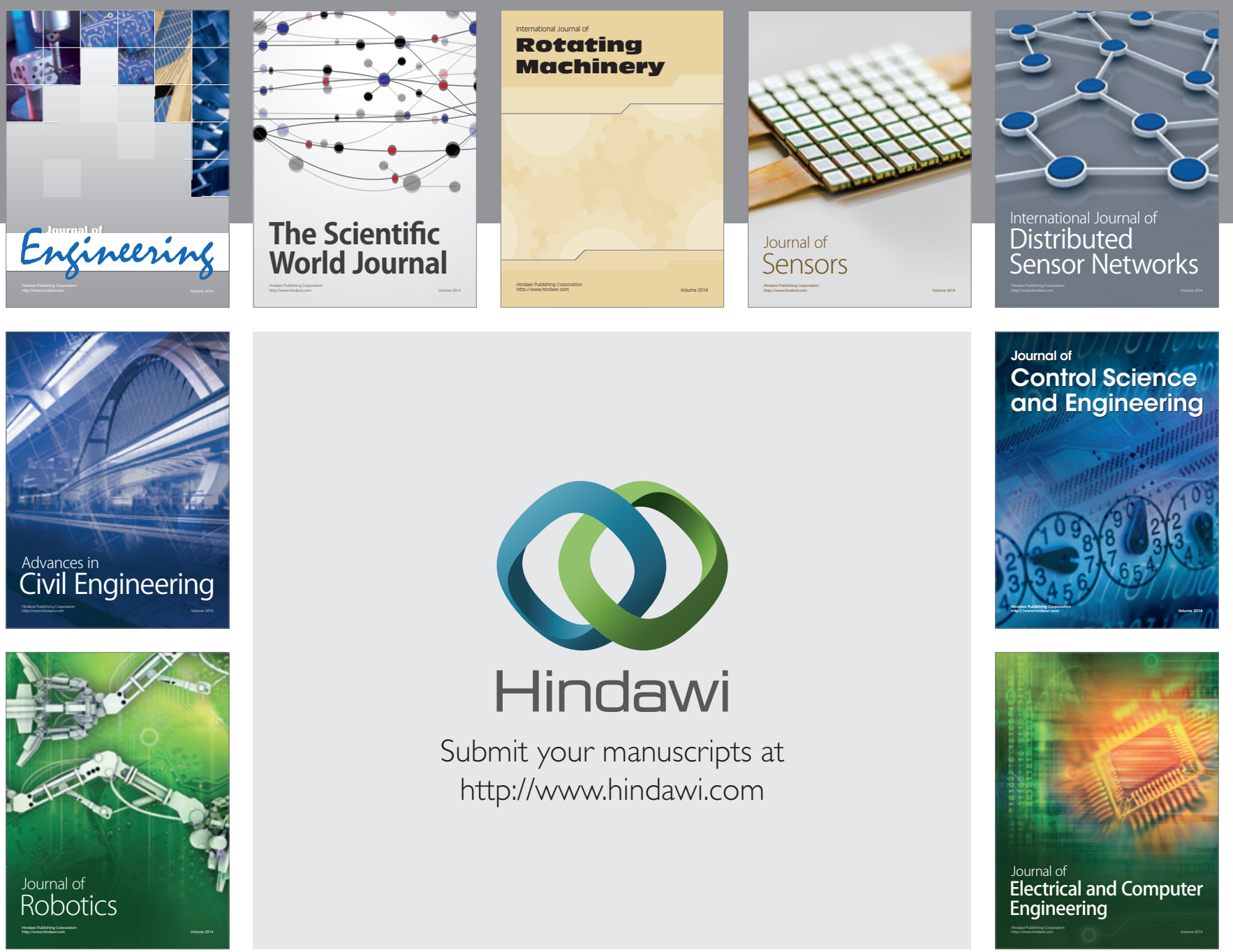

Submit your manuscripts at

http://www.hindawi.com
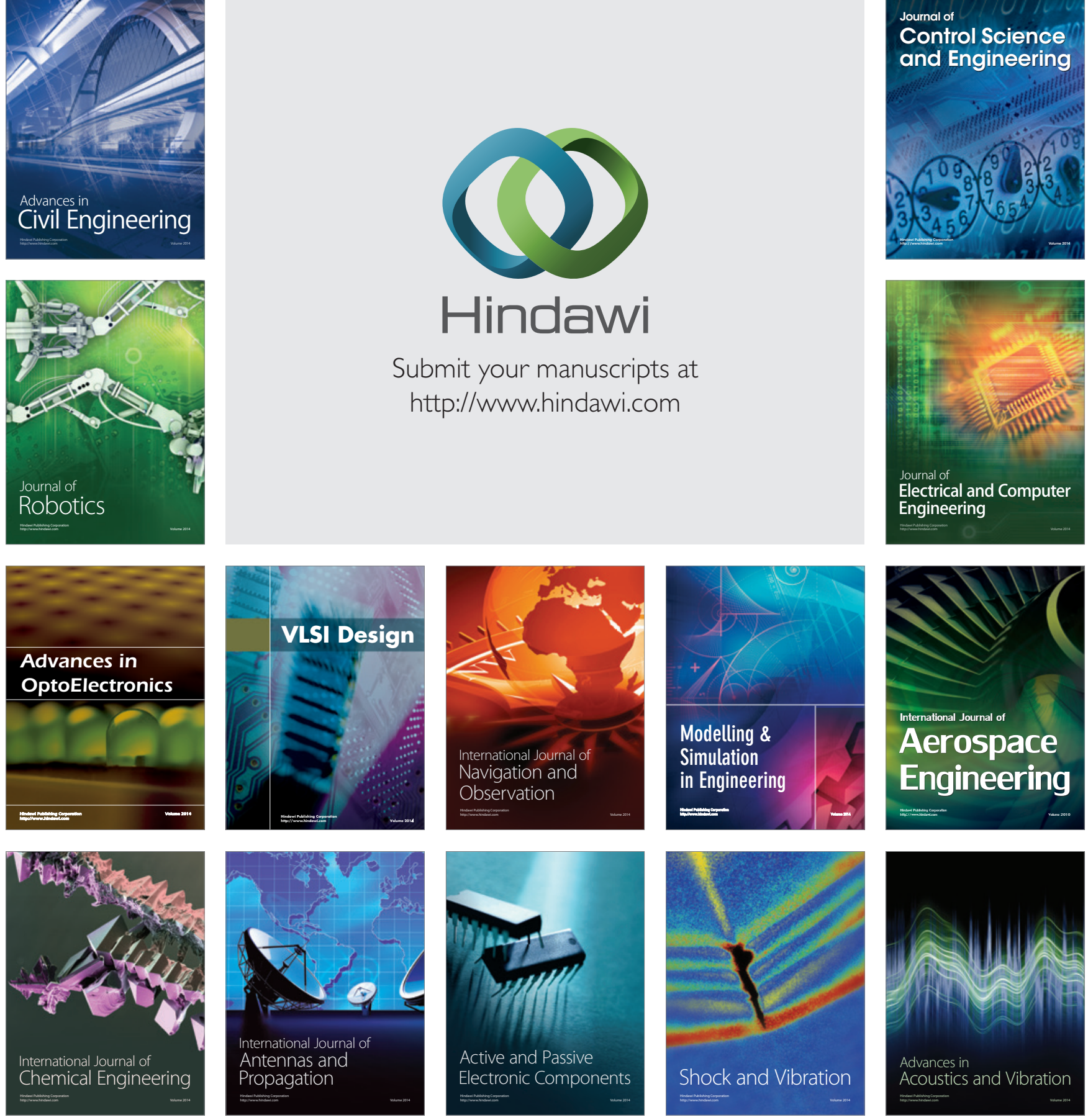